\title{
Diseño de un plan estratégico de investigación y extensión para la Escuela de Computación del TEC
}

\section{Design of a research and extension strategic plan for the School of Computing at TEC}

César Garita-Rodríguez ${ }^{1}$, Mauricio Arroyo-Herrera², Manuel González-Espinoza ${ }^{3}$

Fecha de recepción: 3 de marzo de 2020

Fecha de aprobación: 18 de noviembre de 2019

Garita-Rodríguez, C; Arroyo-Herrera, M; González-Espinoza, M. Diseño de un plan estratégico de investigación y extensión para la Escuela de Computación del TEC. Tecnología en Marcha. Vol. 34-1. Enero-Marzo 2021. Pág 80-91.

doi) https://doi.org/10.18845/tm.v34i1.4732

Doctor en Ciencias de la Computación. Escuela de Computación, Instituto Tecnológico de Costa Rica, Costa Rica. Correo electrónico: cesar@itcr.ac.cr (iD https://orcid.org/0000-0003-4592-3266

2 Ingeniero en Computación. Escuela de Computación, Instituto Tecnológico de Costa Rica, Costa Rica. Correo electrónico: marroyo@tec.ac.cr (D) https://orcid.org/0000-0001-6632-4831

3 Economista. Oficina de Planificación, Instituto Tecnológico de Costa Rica, Costa Rica. Correo electrónico: mangonzalez@tec.ac.cr 


\title{
Palabras clave
}

Plan estratégico; investigación; extensión; computación; universidad.

\begin{abstract}
Resumen
Actualmente las carreras de Computación en la Educación Superior enfrentan retos importantes relacionados con el ejercicio de la investigación y la extensión, como parte de las actividades sustantivas de toda universidad para responder a las necesidades sociales. Estos retos incluyen, por ejemplo, el desarrollo de competencias del recurso humano, la atracción de fondos y la creación de redes de colaboración internacionales. Para responder a ellos, es fundamental para las escuelas o unidades académicas contar con un plan estratégico de investigación y extensión, que contenga las principales metas, actividades e indicadores, para un periodo establecido. En este artículo se describe en detalle la metodología aplicada exitosamente por la Escuela de Computación del Instituto Tecnológico de Costa Rica en el diseño de un plan estratégico quinquenal de investigación y extensión de forma eficaz y con resultados concretos. Esta metodología permitió trabajar en el diseño de manera democrática y acorde con el órgano consultivo, mediante la organización de un conjunto de etapas y actividades definidas, y puede resultar útil como referencia para otras escuelas de computación en Latinoamérica.
\end{abstract}

\section{Keywords}

Strategic planning; research; extension; university; computing school.

\begin{abstract}
Nowadays, computing programs in higher education face important challenges related to research and extension exercise, as part of the substantive activities of every university, including for example: the development of competencies by its employees, fundraising, and the creation of international collaborative networks. In order to respond to these challenges, it is essential for the schools or the academic divisions to have a strategic research and extension plan that contains the main goals, activities, and indicators to be fulfilled within a given period. This article describes in detail the methodology successfully applied by the Computing School of the Costa Rican Institute of Technology (TEC), in the design of a five-year strategic plan for research and extension, in a relatively short time. This methodology involved participatory and purposeful work, through well-defined stages and activities; it could be useful to take it as reference for other computer schools in Latin America.
\end{abstract}

\section{Introducción}

La Escuela de Ingeniería en Computación del Instituto Tecnológico de Costa Rica (TEC) fue creada en 1976 originalmente con el "propósito de formar profesionales con capacidades y habilidades para administrar la función de los sistemas de información en las empresas y desarrollar sistemas de información administrativos" [1]. En la actualidad, la Escuela tiene la misión de contribuir a la sociedad costarricense en la generación de conocimiento científicotecnológico en computación por medio de la docencia, la investigación, la extensión y la vinculación externa, con base en principios de excelencia académica, pertinencia social, regionalización, equidad y formación integral [2].

La Escuela ofrece el plan de estudios de Ingeniería en Computación en los cinco campus o sedes del TEC y en total atiende a una población de alrededor de 1500 estudiantes. Además, 
junto con otras escuelas participa en la ejecución de otros programas académicos de grado como Ingeniería en Computadores y Administración de Tecnologías de Información.

En cuanto a programas de posgrado, desde 1986 la Escuela imparte la Maestría en Computación, que actualmente ofrece dos opciones: Ciencias de la Computación y Gerencia de Tecnologías de Información [3]. En la última década, la Escuela ha participado en la ejecución de programas de maestría y doctorado junto con otras Escuelas, a saber: la Maestría en Gerencia de Proyectos, el Doctorado Interuniversitario en Ciencias Naturales para el Desarrollo y el Doctorado en Ingeniería.

Además, cuenta con un Centro de Investigaciones en Computación creado en 1992, que tiene como propósito contribuir a la solución de problemas mediante la generación, adaptación, incorporación y difusión de conocimientos informáticos. En particular, esta unidad debe promover en forma determinada la investigación y la extensión por parte de la Escuela.

Para realizar las labores derivadas de la ejecución de sus planes de estudio, investigación y extensión, la Escuela cuenta con alrededor de sesenta profesores a tiempo completo y unos treinta a tiempo parcial.

A partir del 2010, la Escuela decidió someterse a procesos continuos de autoevaluación y acreditación por medio del SINAES (Sistema Nacional de Acreditación de la Educación Superior). Es así que a la fecha se han acreditado los programas de Ingeniería en Computación y de la Maestría en Computación. Este proceso ha implicado necesariamente el establecimiento de compromisos de mejora que deben ser verificados ante el ente acreditador, el cual realiza inspecciones cada cuatro años a partir de informes de cumplimiento bianuales.

En este contexto de autoevaluación y acreditación de la calidad, y como resultado de la autoevaluación de la carrera de Ingeniería en Computación y de los programas de Maestría en Computación, se estableció que como parte de la mejora del proceso educativo y propiamente en lo referente a investigación se debía desarrollar un plan estratégico de investigación y extensión. . Los principales aspectos que mejorar identificados en estas autoevaluaciones fueron la participación de un reducido grupo de docentes en investigación, la percepción por un porcentaje significativo de los docentes de que la carrera no presentaba oportunidades para realizar investigación y que no había grupos de investigación formalmente constituidos dentro de los programas. Por otro lado, con respecto a la extensión, los hallazgos incluyeron la ausencia de un plan estratégico específico y el hecho de no contarse con un registro de los resultados de las acciones de extensión.

En el plano institucional de mejoramiento de la calidad, el TEC se destacó en el 2017 como la primera universidad del continente americano que logró obtener la acreditación institucional otorgada por Francia por medio de HCERES (Alto Consejo de Evaluación de la Investigación y la Educación Superior de Francia) [4]. El informe institucional derivado de esta acreditación indicó algunas debilidades en la temática de investigación, a saber: la falta de cultura de investigación en la institución, un rendimiento débil en términos de número de publicaciones en revistas internacionales, la dispersión estructural de entidades de investigación y a proporción relativamente pequeña de miembros del personal académico con grado de doctorado.

En este contexto, teniendo presentes los compromisos de los procesos de acreditación y el planteamiento estratégico, la Escuela de Computación ha venido direccionando sus esfuerzos al desarrollo del plan estratégico de investigación y extensión (PEIE), con el propósito de coadyuvar a la producción científica y social. Al mismo tiempo, estos esfuerzos han sido relevantes para mantener la acreditación de los programas de la Escuela ante el SINAES y contribuir a resolver las carencias indicadas en el proceso de acreditación institucional ante HCERES. 
Además, cabe mencionar que se ha procurado alinear el PEIE con el Plan Estratégico General de la Escuela, establecido para el periodo 2017- 2022. En particular, el plan general incluye un eje de investigación, extensión y acción social, con líneas estratégicas que han sido consideradas dentro de la estructuración del PEIE. El plan general fue diseñado con éxito siguiendo una metodología muy similar a la que se describe en este trabajo.

El presente artículo describe la metodología y el resultado del proceso de diseño del PEIE para la Escuela de Computación. El proceso seguido se considera valioso en vista de que el resultado se alcanzó en un corto tiempo y con resultados muy satisfactorios. Se puede adelantar que el trabajo en talleres fue un medio importante para extraer y sintetizar la información relevante en la construcción del plan.

\section{Trabajos relacionados}

En América Latina, la planificación estratégica como forma de orientar la vida universitaria ha significado para las universidades a lo largo de su historia grandes retos en procura de adaptarse a entornos en constante evolución. Las comunidades universitarias han articulado importantes esfuerzos colectivos para enfrentar los cambios que la sociedad demanda. Berheim señala que "la preocupación de las universidades latinoamericanas por extender su acción más allá de los linderos académicos arranca de la Reforma de Córdoba de 1918" [5].

En este mundo altamente integrado, se requiere capacidad para gestionar los cambios internos que las comunidades universitarias requieren. Por esta razón, aplicar metodologías de planificación estratégica en la gestión del quehacer universitario y en particular, en investigación y extensión toma gran importancia, pues en áreas altamente especializadas como la computación, la complejidad de los desarrollos tecnológicos genera rápidas transformaciones.

Trabajos relacionados, como el de Davara [6], demuestran que las herramientas de planificación estratégica efectivamente deben actualizarse y funcionar en un mundo en extremo complejo y cambiante; sin embargo, indican que principalmente deberá tenerse en cuenta a las personas que participan en el proceso, dado que de sus conocimientos, habilidades, fuentes de datos e interrelaciones dependerá el alcanzar con éxito las metas del plan. Pulgarin y Rivera [7] así como Martelo et al. [8] agregan que hoy existe una gran cantidad de herramientas para apoyar las formas de realizar planificación estratégica, que permite a las organizaciones orientar su toma de decisiones.

En este contexto, el TEC ha llevado a cabo múltiples procesos de planificación estratégica desde un enfoque de mejora continua, incluyendo el Plan Estratégico Institucional 2017 - 2021 , así como diferentes microprocesos de planificación (desarrollados en diferentes unidades académicas), que responden tanto a objetivos de acreditación como a actividades de gerencia interna para la gestión administrativa cotidiana de las unidades. Estas experiencias han permitido el crecimiento de las ventajas comparativas de la Institución con respecto a otras universidades, que a su vez le han valido la acreditación internacional por parte de HCERES, como se mencionó en la sección anterior.

En el caso particular del proceso de construcción del PEIE en el área de Investigación de la Escuela de Ingeniería en Computación, se trabajó bajo un enfoque multicriterio para la toma de decisiones utilizando diferentes herramientas de la planificación estratégica: análisis FODA, técnicas de priorización, análisis MECA, técnicas de categorización, análisis de brechas y análisis gráfico [9]; la Oficina de Planificación (OPI), del Instituto Tecnológico de Costa Rica (TEC), brindó el acompañamiento necesario para ello.

Gracias a los profesionales expertos en planificación, fue posible desarrollar el PEIE en la Escuela, pues "la conducción de un ejercicio de planificación estratégica no se puede aprender en los 
libros" [10]. Justamente, la forma de trabajo articulado y participativo entre colaboradores de la OPI y profesores de la Escuela probó ser uno de los aspectos innovadores de la metodología aplicada y responde a la tendencia global de encontrar modelos participativos y dinámicos acordes a las necesidades particularidades de las instituciones de Educación Superior.

En ciertos casos, se aplicaron también técnicas computacionales relacionadas con cursos de "Arquitectura de la Información" y "Calidad del Software" para apoyar en la resolución de problemas específicos, como se explicará en la sección "Definición de líneas estratégicas".

Otro rasgo característico de la metodología implementada es que se basa en la concepción de la planificación estratégica "como una hoja de ruta que orientará el camino y el devenir de una organización a largo plazo, diseñada por medio de la búsqueda del equilibrio entre la misión y la visión, el qué y el para qué, las fortalezas y debilidades deducidas del entorno interno y las amenazas y oportunidades del externo" [6].El proceso de planificación estratégica siguió un avance gradual, de lo general hacia lo específico, hasta construir los insumos que han permitido a la organización tomar decisiones en el área de interés. En este proceso, se partió de una visualización del estado actual de la investigación y la extensión; se planteó el futuro deseable y se definieron los objetivos y las estrategias para lograrlos, de forma colaborativa. Esto resultó ventajoso, pues permitió el empoderamiento de la comunidad y su participación en el proceso, construyendo las metas sobre la idiosincrasia y formas de trabajo propias del área profesional específica.

En resumen, la metodología aplicada en este trabajo constituye una base de referencia para sistematizar de forma cada vez más robusta los procesos de planificación estratégica en las universidades e instituciones dirigidas a promover la ciencia y la tecnología, y en particular, a fortalecer su vínculo con la sociedad latinoamericana por medio de la investigación y la extensión.

\section{Metodología general}

Como se explicó anteriormente, la Escuela de Computación acordó diseñar un plan estratégico de investigación y extensión mediante la aplicación formal de una metodología ágil y participativa. Se nombró una comisión integrada por miembros del Consejo de Escuela para coordinar las tareas por realizar para el diseño del plan. En diferentes etapas de su desarrollo, también participaron otros miembros de la Escuela, incluidos los coordinadores de unidades, y representantes de investigadores docentes de diferentes sedes. A lo largo del proceso, se contó con el apoyo fundamental de la Oficina de Planificación del TEC mediante su asesoría técnica y logística.

Antes de definir las etapas específicas del diseño, se definió el objetivo general del plan y se llevó a cabo un análisis previo de documentos institucionales y de la Escuela que podían servir como insumos al plan. A continuación, se describen el objetivo general, el análisis del contexto y las fases en el desarrollo de la metodología.

\section{Objetivo general}

Para el Plan Estratégico de Investigación, Extensión y Acción Social 2019-2023 se estableció el siguiente objetivo general:

"Diseñar un modelo de organización orientada a la investigación, la extensión, la acción social y la vinculación de la Escuela, conforme a principios de excelencia, pertinencia y regionalización". 


\section{Análisis del contexto}

Como parte de un análisis de contexto para el desarrollo del Plan Estratégico de Investigación y Extensión (PEIE) para la Escuela de Computación, se llevó a cabo una revisión de documentos que representaban insumos importantes para su diseño. Entre otros, los siguientes documentos fueron estudiados para ese propósito:

- Plan Estratégico (PE) de la Escuela de Computación 2017-2022 y Plan Estratégico del TEC 2017-2021.

- Compromiso de mejoras para la acreditación de los programas de Bachillerato y Maestría en Computación.

- Lineamientos internos para investigación y extensión en el TEC.

- Informe de Acreditación Institucional con el Alto Consejo de Evaluación de la Investigación y la Educación Superior, de Francia (HCERES).

- Ejes de Conocimiento Estratégicos y Modelo Académico del TEC.

El resultado del análisis de contexto se resumió en un documento que fue distribuido entre los participantes del diseño del plan.

\section{Talleres y fases de la metodología}

Para el desarrollo del PEIE, se estableció trabajar en una metodología desarrollada por etapas mediante talleres de trabajo colaborativo, según la figura 1.

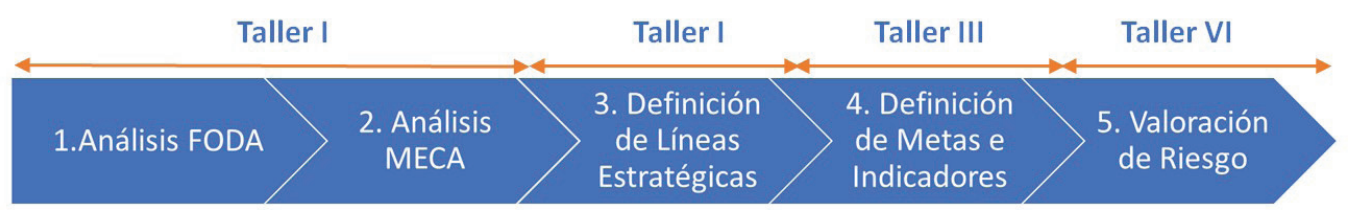

Figura 1. Metodología propuesta para diseñar el plan.

Como puede observarse en la figura, para la ejecución de las etapas se organizaron cuatro talleres de trabajo en los que participaron diferentes representantes de la Escuela (invitados según la naturaleza de cada etapa). En total, participaron alrededor de treinta personas durante todo el proceso.

Antes de cada etapa también se realizaron actividades de planeamiento, organización, procesamiento de información, realización de informes y ajustes con base en retroalimentación recibida por parte de los participantes.

El desarrollo de los talleres y las etapas se describe en detalle en las siguientes secciones:

\section{Taller I - FODA Y MECA}

Como primer taller se realizó un diagnóstico relacionado con los procesos de investigación y extensión en la Escuela, utilizando la herramienta FODA (fortalezas, oportunidades, debilidades y amenazas), y luego, un análisis MECA en el que se identificaron acciones para mejorar las fortalezas (M), explotar las oportunidades (E), contrarrestar las debilidades (C) y afrontar las amenazas (A). En este taller participaron cerca de veinticinco personas como representantes de los programas y sedes involucrados. Las actividades y productos de estos análisis se describen a continuación. 


\section{Análisis FODA}

La realización del FODA comprendió las siguientes actividades:

- Conformación de grupos de trabajo: Se formaron aleatoriamente cuatro grupos de trabajo. A cada grupo se le asignó una letra del FODA.

- Lluvia de ideas por cada letra del FODA: Cada grupo realizó una lluvia de ideas sobre las variables más importantes relacionadas con la inicial de la palabra correspondiente, respecto a temas de investigación, extensión y acción social, en la Escuela.

- Integración de variables internas y externas: Se reunieron los grupos de acuerdo con el tipo de las variables que les fueron asignadas: las internas (fortalezas, debilidades) y las externas (oportunidades, amenazas), las discutieron y seleccionaron las diez más relevantes por cada letra. Cada grupo anotó las variables seleccionadas en una cartulina y la colocó en la pared de la sala de reunión.

- Priorización del FODA: Por medio de votación con puntos [11], todos los participantes votaron individualmente para seleccionar las tres ideas de mayor relevancia (con 3 como el puntaje más alto; 2, el segundo en importancia, y 1, el menor) por cada variable anotada. Para esto, a cada participante se le dieron tres papeles adhesivos, uno por cada puntaje, por cada cartulina, con el propósito de que asignara los puntajes según su valoración.

- Consolidación del FODA: Finalmente, se escogieron las cinco variables de cada letra con los puntajes más altos.

El producto de esta etapa fueron listas de enunciados relacionados con fortalezas, oportunidades, debilidades y amenazas identificadas y priorizados de manera grupal por los participantes. En la figura 2 se incluye un ejemplo de los factores identificados por cada dimensión del análisis FODA.

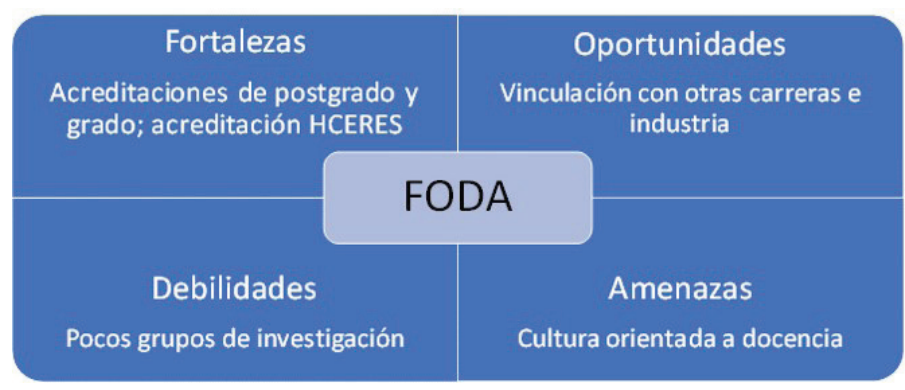

Figura 2. Análisis FODA.

\section{Análisis MECA}

La realización del MECA comprendió las siguientes actividades:

- Análisis MECA: Se mantuvieron los cuatro grupos anteriores y se detallaron las acciones que indica el MECA, para cada una de las variables del FODA, a modo de respuestas a las preguntas ¿cómo mejorar las fortalezas?, ¿cómo explotar las oportunidades?, ¿cómo contrarrestar las debilidades?, ¿cómo afrontar las amenazas? Cada grupo escribió en una cartulina las acciones que consideró más importantes en relación con cada variable del FODA.

- Validación del análisis: Cada grupo expuso las acciones propuestas para llevar a cabo el MECA. Se discutieron en pleno con base en cada presentación y se realizaron ajustes de forma consensuada. 
Como resultado del MECA se obtuvieron listas de acciones identificadas por cada letra MECA correspondiente a cada letra FODA. En la figura 3. , se incluye un ejemplo de los factores MECA correspondientes a los factores FODA antes señalados.

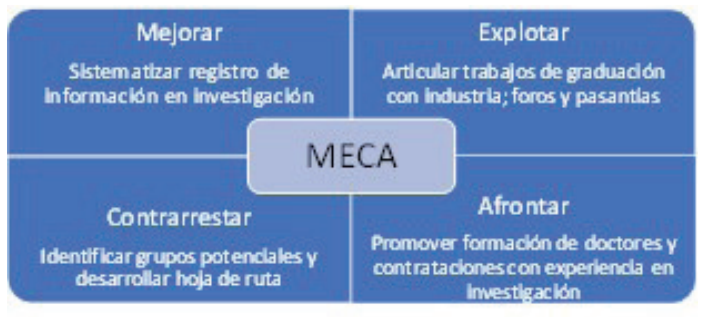

Figura 3. Factores MECA.

\section{Taller II - Definición de Líneas Estratégicas}

Con base en los análisis FODA y MECA, en el segundo taller se propusieron líneas estratégicas para agrupar las acciones identificadas en el MECA. El producto de esta fase fue una lista de líneas estratégicas de investigación y extensión.

Para lograr el objetivo de este taller, se modeló el problema de identificar las líneas estratégicas como un problema de ordenamiento de tarjetas abierto (open card sorting [12]). Para ello, cada acción de las listas obtenidas del análisis MECA se escribió en una tarjeta. Las tarjetas fueron distribuidas entre los participantes del taller para que las agruparan y asignaran un nombre a cada grupo. Los grupos se convirtieron en líneas estratégicas. Es decir, las líneas estratégicas se visualizan como agrupamientos conceptuales de las acciones identificadas en el MECA.

Además de identificar las líneas estratégicas, se hizo un ejercicio de valoración por parte de los participantes, para contrastar el nivel de madurez actual y el nivel de madurez deseado para cada línea. Esta valoración puede usarse como insumo para luego calendarizar las acciones específicas del plan.

En resumen, las actividades realizadas en este taller fueron las siguientes:

- Repaso de resultados de MECA: Se realizó una exposición en la que se resumieron las actividades y resultados de la fase anterior.

- Conformación de grupos de trabajo: Se dividieron los participantes en cuatro grupos de trabajo (uno por cada letra MECA), de forma aletaoria.

- Definición de líneas estratégicas: Cada grupo identificó las líneas estratégicas correspondientes, haciendo un ejercicio de agrupamiento de tarjetas (acciones MECA). Para ello, se facilitó a cada grupo un juego completo de las tarjetas de todas las acciones MECA y se les solicitó que definieran alrededor de 7 grupos (categorías que agruparan las tarjetas) y le dieran un nombre a cada grupo.

- Exposición de resultados por grupo: Cada grupo presentó los resultados de la identificación de líneas. Para este propósito, los grupos anotaron los nombres de las líneas en hojas respectivas, y en cada hoja pegaron las tarjetas correspondientes. Las hojas fueron pegadas a su vez en rotafolios que se colocaron alrededor del espacio de trabajo para facilitar la exposición e incentivar la validación por los participantes.

- Unificación de resultados: Para conjuntar los resultados, se nombró una mesa de unificación con un representante de cada grupo para llegar a un consenso respecto a la definición final de las líneas estratégicas. 
- Valoración de madurez de líneas estratégicas: Una vez identificadas y unificadas las líneas estratégicas, los participantes valoraron el nivel de madurez actual y el nivel de madurez deseado para cada línea. En este caso, se utilizó como referencia el modelo de madurez CMMI (Capability Maturity Model Integration [13]) y cada participante valoró de 1 a 5 el nivel de madurez de cada línea (1: Inicial; 2: Administrado; 3: Definido; 4: Administrado cuantitativamente; 5: Optimizado). Con base en la valoración, se calculó el promedio de madurez del estado actual y del estado deseado para cada línea estratégica.

Como producto de este taller, se identificaron las líneas estratégicas del plan, que se ilustran en la figura 4.

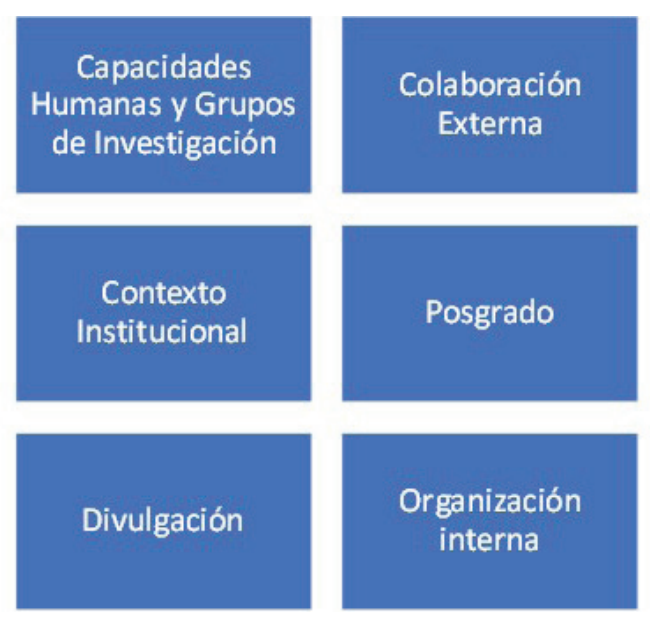

Figura 4. Líneas estratégicas del Plan.

\section{Taller III - Definición de Metas e Indicadores}

El taller III tuvo como objetivo principal definir las metas e indicadores del plan. Para ello se contó con una plantilla (hoja de cálculo estructurada) sugerida por los asesores de la OPI para la elaboración detallada de las metas e indicadores, con base en ejes, líneas y acciones del plan. Además, como trabajo previo al taller se realizó una propuesta para vincular las líneas estratégicas resultantes de la fase anterior en función de las líneas del Plan Estratégico de la Escuela (aquí denominadas "ejes"). Es decir, las líneas del PEIE fueron incorporadas dentro de las líneas del Plan Estratégico de la Escuela como marco superior.

El tercer taller consistió en completar de forma colaborativa y participativa la plantilla facilitada por la OPI para la definición de las metas e indicadores.

En particular, las actividades llevadas a cabo fueron las siguientes:

- Repaso de los resultados de identificación de líneas estratégicas: Se realizó una exposición en la que se resumieron las actividades y resultados de la fase anterior.

Presentación de la metodología: Se expuso la metodología que seguir en este taller con apoyo en la plantilla para la elaboración de metas e indicadores proporcionada por la OPI. Se presentaron sugerencias sobre cómo definir las metas y los indicadores.

- Conformación de grupos de trabajo: Se dividieron los participantes en dos grupos de trabajo, a cada uno de los cuales se les asignaron tres líneas estratégicas. 
- Definición de indicadores y metas: Cada grupo llenó de manera colaborativa la plantilla proporcionada para la definición de las metas e indicadores, con base en las líneas correspondientes.

- Exposición de resultados por grupo: Cada grupo presentó las definiciones de metas e indicadores; estas se analizaron y discutieron. Se realizaron ajustes según la valoración de los participantes.

Los resultados quedaron escritos en las plantillas; cada una incluyó la siguiente información, referente a una acción MECA:

- Eje del Plan Estratégico de la Escuela

- Línea estratégica

- Acción MECA

- Metas

- Actividades

- Indicadores

- Responsables

- Plazos

- Requerimientos

En el cuadro 1 se presentan, como ejemplo, las metas, actividades e indicadores correspondientes a la acción "Identificar grupos potenciales y desarrollar hoja de ruta".

Cuadro 1. Ejemplo de metas e indicadores.

\begin{tabular}{|c|c|c|}
\hline Metas & Actividades & Indicadores \\
\hline $\begin{array}{c}\text { Promover al menos seis grupos } \\
\text { de investigación y extensión } \\
\text { formales, con criterios de calidad. }\end{array}$ & $\begin{array}{c}\text { - Elaborar un mecanismo para } \\
\text { identificar grupos de investigación } \\
\text { con base en criterios de calidad. }\end{array}$ & $\begin{array}{c}\text { Al menos seis grupos de investigación } \\
\text { consolidados según criterios de } \\
\text { calidad, incluyendo al menos un grupo } \\
\text { por unidad académica. }\end{array}$ \\
& - Elaborar hoja de ruta para \\
consolidar cada grupo. & - Promover a los grupos \\
& identificados según hoja de ruta. & \\
\hline
\end{tabular}

Taller IV - Valoración del Riesgo

En esta última etapa se realizó una identificación y valoración de riesgos que podían obstaculizar el cumplimiento de las metas establecidas en el plan. El producto de esta etapa fue un conjunto de riesgos y su valoración en función de las metas establecidas. Las actividades realizadas en este taller final se resumen a continuación:

1. Repaso de resultados de la identificación de metas e indicadores: Se realizó una exposición en la que se resumieron las actividades y resultados de la fase anterior.

2. Presentación de metodología: Se expuso la metodología por seguir en este taller con apoyo en la plantilla para valoración del riesgo institucional proporcionada por la OPI. Se presentaron el contexto y los conceptos fundamentales asociados con la valoración del riesgo institucional. Se dieron sugerencias sobre cómo llenar la plantilla, para lo cual se les facilitó a los participantes un catálogo de riesgos institucionales que habían sido previamente identificados por la OPI en diferentes proyectos institucionales, para que 
fuesen tomados como referencia. Se les recomendó proponer como base un riesgo que el grupo considerara como el principal para cada meta.

3. Conformación de grupos de trabajo: Se dividieron los participantes en dos grupos de trabajo. Se estableció que cada grupo valoraría las metas previamente establecidas para tres líneas estratégicas. Cada grupo llenó de manera colaborativa las plantillas proporcionadas para las metas correspondientes.

4. Exposición de resultados por grupo: Cada grupo presentó los resultados de la valoración del riesgo para las metas respectivas. Se analizaron y discutieron los resultados. Se realizaron ajustes según la valoración de los participantes.

Como resultado de esta etapa, se obtuvo una plantilla con esta información por cada meta del plan:

- Identificación del riesgo (evento de riesgo)

- Calificación del riesgo (probabilidad, impacto)

- Evaluación del control (posible forma de control)

- Acciones para mitigar el riesgo

A continuación, en el cuadro 2 se presenta un ejemplo de la valoración de riesgo para una meta particular.

Cuadro 2. Ejemplo de valoración del riesgo.

\begin{tabular}{|c|c|c|c|}
\hline Meta & Evento & Calificación & Acciones de mitigación \\
\hline $\begin{array}{c}\text { Promover al menos seis grupos de } \\
\text { investigación y extensión formales } \\
\text { con criterios de calidad. }\end{array}$ & $\begin{array}{c}\text { Falta de consenso en la } \\
\text { definición de los criterios } \\
\text { para identificar a los grupos. }\end{array}$ & Muy alto & $\begin{array}{c}\text { Hacer conciencia del } \\
\text { por qué y para qué de } \\
\text { los grupos. }\end{array}$ \\
\hline
\end{tabular}

\section{Conclusiones y trabajo futuro}

Como principal conclusión a la realización del diseño del PEIE siguiendo la metodología presentada en este artículo se tiene el contar actualmente con el Plan de Investigación y Extensión para los próximos cinco años, como una propuesta que incluye los siguientes elementos principales:

- Definición de líneas estratégicas

- Especificación de acciones MECA con base en análisis FODA

- Metas bien definidas

- Actividades para alcanzar las metas

- Indicadores para medir el logro de las metas

- Definición de los responsables de las metas

- Plazos para la realización de las actividades

- Valoración de los riesgos asociados a las metas y acciones para contrarrestarlos.

La metodología seguida permitió la realización de las actividades de forma democrática, participativa y guiada, con actores de toda la Escuela; todo ello de una manera ágil y en corto tiempo. 
Además de técnicas formales de planificación estratégica, se aplicaron y adaptaron también técnicas o modelos computacionales derivados de temas programáticos de los cursos de la Escuela, tales como ordenamiento de tarjetas y el modelo de madurez CMMI (ver sección V).

La asesoría y apoyo técnico brindado por la Oficina de Planificación fue fundamental para poder realizar esta propuesta de plan. De hecho, con base en la experiencia y los resultados obtenidos, se recomienda tomar como base la metodología de planificación utilizada, para la realización de futuras actividades similares en la Escuela.

La propuesta de plan ya fue sometida a consideración del Consejo de Escuela para conseguir su aprobación oficial. Como trabajo futuro, una vez aprobada, sería conveniente elaborar un mapa de ruta con base en los productos del plan, que facilite la gestión eficaz de las actividades y las metas propuestas para los próximos años.

Finalmente, la metodología utilizada puede ser útil como referencia para otras escuelas de computación en América Latina, que de manera similar necesiten desarrollar procesos de planeación estratégica relacionados con investigación y extensión, o bien con otras actividades académicas o administrativas.

\section{Agradecimientos}

Los autores desean agradecer a los miembros de la Escuela de Computación que participaron en los talleres de trabajo, así como a los compañeros de la Oficina de Planificación que dieron su asesoría y seguimiento durante todo el proceso de diseño del plan estratégico presentado en este artículo.

\section{Referencias}

[1] TEC, "Instituto Tecnológico de Costa Rica (TEC)", https://www.tec.ac.cr/, 2019.

[2] TEC, "Escuela de Ingeniería en Computación", https://www.tec.ac.cr/escuelas/escuela-ingenieria-computacion, 2019.

[3] TEC, "Programa de Maestría en Computación", https://www.tec.ac.cr/programas-academicos/maestria-computacion, 2019.

[4] TEC, "TEC, primera universidad latinoamericana acreditada por Consejo Francés de Evaluación HCERES", https://www.tec.ac.cr/hoyeneltec/, 2017.

[5] C. T. Berheim, "El nuevo concepto de extensión universitaria y difusión cultural y su relación con las políticas de desarrollo cultural en América Latina", en Anuario de Estudios Centroamericanos, pp. 93-126, 1978.

[6] F. Davara, "Planificación estratégica e inteligencia económica: herramientas de gestión del cambio", Cuadernos de Estrategia, vol. 174, pp. 189-229, 2015.

[7] S. Pulgarin y H. Rivera, "Las herramientas estratégicas: un apoyo al proceso de toma de decisiones gerenciales (Strategic tools: Supporting the managerial decision making process)", Criterio Libre, vol. 10, pp. 89-114, 2012.

[8] R. Martelo, A. Ponce y F. Acuña, "Guía metodológica para el diseño de un plan estratégico informático en instituciones de Educación Superior", Formación Universitaria, vol. 9, 2016.

[9] Ž. Živković, D. Nikolić, M. Savić, P. Djordjević, and I. Mihajlović, "Prioritizing strategic goals in Higher Education organizations by using a SWOT-PROMETHEE/GAIA-GDSS model”, Group Decision and Negotiation, vol. 26, pp. 829-846, 2017.

[10] F. J. García-Peñalvo, "Dirección Estratégica”, https://repositorio.grial.eu/handle/grial/1168, 2018.

[11] J. Dalton, "Dot voting", in Great Big Agile: An OS for agile leaders [formato electrónico]. Apress, 2019.

[12] P. Morville, L. Rosenfeld, and J. Arango, Information architecture: For the Web and beyond, 4th ed. [formato electrónico]. O’Reilly Media, 2015.

[13] C. Institute, "CMMI Institute", https://cmmiinstitute.com/, 2019. 